\title{
Lombalgia ocupacional e a postura sentada*
}

\author{
Occupational low back pain and the sitting position
}

Suélem Silva de Barros ${ }^{1}$, Rita di Cássia de Oliveira Ângelo², Érica Patrícia Borba Lira Uchôa ${ }^{3}$

* Recebido da Universidade Católica de Pernambuco. Recife, PE.

\section{RESUMO}

JUSTIFICATIVA E OBJETIVOS: A lombalgia é frequente na população economicamente ativa, podendo estar associada à determinadas atividades ocupacionais em que o trabalhador permanece sentado em condições antiergonômicas por tempo prolongado. O objetivo deste estudo foi investigar a provável relação existente entre lombalgia e atividades laborais executadas na posição sentada.

MÉTODO: Estudo analítico transversal, realizado em uma instituição de ensino superior da cidade de Recife, incluindo 239 funcionários do corpo técnico administrativo. Foram coletados dados relativos às características sócio-demográficas e antropométricas; avaliação da percepção dolorosa pela escala de dor autopercebida; avaliação da percepção da dor lombar, limitações e dificuldades na execução das atividades laborais e de vida diária, por meio dos questionários de avaliação funcional de Oswestry e de Roland-Morris. Em seguida, foi aplicado o teste físico Screening da Polestar Education ${ }^{\circledR}$ para avaliação do nível de força dos músculos estabilizadores do tronco e da amplitude de movimento da coluna vertebral.

RESULTADOS: Os dados sugerem correlação significativa entre atividades laborais executadas na posição sentada e lombalgia. A prevalência de dor lombar crônica foi de $95,2 \%$, sendo maior nos funcionários com

1. Fisioterapeuta, em Fisioterapia Traumato-Ortopédica e em Saúde Pública. Recife, PE, Brasil.

2. Fisioterapeuta, Professora do Curso de Fisioterapia da Universidade de Pernambuco (UPE). Petrolina, PE, Brasil.

3. Fisioterapeuta, Professora do Curso de Fisioterapia das Faculdades Integradas do Recife (FIR) e da Universidade Católica de Pernambuco (UNICAP). Recife, PE, Brasil.

Endereço para correspondência:

Suélem Silva de Barros

Rua Silvino Lopes, 92 - Cajá.

55610-090 Vitória de Santo Antão, PE.

Fone: (081) 8846-7601

Email: suelem.barros@yahoo.com.br idade superior a 40 anos, sedentários e que trabalham há mais tempo na instituição. As variáveis: sexo, índice de massa corpórea e realização de pausas durante a jornada de trabalho não se comportaram como fator de risco para o aparecimento dos sintomas.

CONCLUSÃO: Os resultados sugerem uma provável associação entre dor lombar e atividades laborais na posição sentada e alertam para a necessidade de cuidados com a saúde da coluna no ambiente de trabalho.

Descritores: Coluna vertebral, Lombalgia, Postura.

\section{SUMMARY}

BACKGROUND AND OBJECTIVES: Low back pain is frequent in the economically active population and may be associated to some occupational activities where the worker remains in the sitting position in anti-ergonomic conditions for a long time. This study aimed at investigating the possible relationship between low back pain and labor activities performed in the sitting position.

METHOD: Transversal analytical study carried out in a university of Recife, including 239 employees of administrative technical areas. Data were collected with regard to socio-demographic and anthropometrical features; pain was evaluated by the self-perceived pain scale; lumbar pain perception and difficulty to perform labor and daily life activities were evaluated by Oswestry and Roland-Morris functional evaluation questionnaires. Then, the Polestar Education's ${ }^{\circledR}$ Screening physical test was applied to evaluate the strength level of body stabilizing muscles and spinal movement amplitude.

RESULTS: Data suggest a significant correlation between labor activities in the sitting position and low back pain. The prevalence of chronic low back pain was $95.2 \%$, being higher in employees above 40 years of age, sedentary and working for the institution for a longer period. Gender, body mass index and pauses during working hours were not risk factors for the appearance of symptoms. 
CONCLUSION: Our results suggest a possible association between low back pain and labor activities in the sitting position and call the attention to the need of care with spinal health in the workplace.

Keywords: Low back pain, Posture, Spine.

\section{INTRODUÇÃO}

A lombalgia é um sintoma de etiologia multifatorial, acomete ambos os sexos e apresenta alta incidência na população economicamente ativa, incapacitando temporária ou definitivamente para execução das atividades profissionais ${ }^{1,2}$. Quando persiste por mais de seis meses é caracterizada como dor crônica, tornando-se um problema de saúde pública por interferir nas relações sociais, econômicas, profissionais e culturais ${ }^{2,3}$. Afeta mais de $70 \%$ dos indivíduos em alguma época de suas vidas ${ }^{4}$. No cenário mundial, apresenta uma prevalência média de $23,5 \%{ }^{2}$.

É grande a diversidade de distúrbios clínicos relacionados à presença de dor lombar. Todavia, sua gênese é frequentemente atribuída à fadiga e às deficiências musculares proporcionadas em posturas inadequadas e repetitivas ${ }^{1,5}$. Alguns autores relatam sua atribuição a determinadas atividades ocupacionais, ressaltando aquelas em que o trabalhador permanece sentado em condições antiergonômicas por tempo prolongado, visto que a manutenção de uma posição fixa não faz parte do modelo anatômico e funcional humano ${ }^{2,5}$.

O homem moderno permanece, em média, um terço da sua vida na postura sentada, o que pode acarretar alterações biomecânicas, como desequilíbrio muscular entre força extensora e flexora do tronco e diminuição da estabilidade e mobilidade do complexo lombo-pelve-quadril, responsáveis pelo desenvolvimento de dores na porção inferior da coluna ${ }^{1,5,6}$. A limitação funcional e as desabilidades laborais geradas pelo desconforto físico, fazem com que a lombalgia seja considerada uma das principais causas de afastamento do trabalho e gastos com despesas médicas, interferindo diretamente na qualidade de vida dos indivíduos sintomáticos ${ }^{4,7}$.

A fisioterapia dispõe de vários recursos que auxiliam no controle do quadro álgico e na re-educação funcional dessas disfunções, promovendo bem estar físico, mental e social e favorecendo o retorno às atividades cotidianas e laborais ${ }^{3,6}$. A identificação da lombalgia e da pré-condição do trabalhador a este sintoma devem fundamentar a atenção básica na implantação de um programa de intervenções terapêuticas e/ou preventi- $\operatorname{vas}^{2,4}$. Questionários validados são descritos como possíveis ferramentas na obtenção de informações relacionadas à biomecânica imposta pela atividade ocupacional e a presença de dor lombar ${ }^{3}$.

Baseado nestas proposições, o objetivo deste estudo foi investigar a relação existente entre dor lombar e atividades laborais executadas na posição sentada.

\section{MÉTODO}

Após aprovação pelo Comitê de Ética da Instituição (CAAE - 0026.0.096.000-08), realizou-se este estudo analítico transversal com 239 funcionários de ambos os sexos, do corpo técnico administrativo de uma instituição de ensino superior da cidade de Recife, que trabalhavam sentados. Foram excluídos os que trabalhavam em postura ortostática, portadores de deficiência física, incapacitados de realizar esforço, gestantes, trabalhadores afastados da empresa no momento da coleta de dados, e os que recusaram participação no estudo.

Todos foram esclarecidos quanto ao objetivo da pesquisa e autorizaram sua participação por meio do termo de consentimento livre e esclarecido. Após a coleta de dados, cada voluntário recebeu um panfleto explicativo contendo orientações e exercícios básicos para a manutenção da saúde da coluna no ambiente de trabalho.

Inicialmente, foi utilizada uma ficha de entrevista, fundamentada em pesquisas já realizadas, para notificação de informações relativas às características sócio-demográficas e antropométricas da população em estudo. As variáveis investigadas foram idade, sexo, massa, altura e índice de massa corporal (IMC). Os indivíduos responderam questões sobre a prática de atividade física, tempo de serviço na instituição e realização de pausas durante a jornada de trabalho, além de perguntas referentes à existência e duração de dor lombar.

A percepção dolorosa foi avaliada pela escala de dor autopercebida com graduação de zero a 10, onde zero representa ausência absoluta de dor e 10 dor máxima. Para averiguar a capacidade dos funcionários em perceber a dor lombar, limitações e dificuldades na execução das atividades laborais e de vida diária foram aplicados os questionários de Avaliação Funcional de Oswestry e de Roland-Morris.

O questionário de Oswestry é uma ferramenta composta por 10 sessões de perguntas constituídas de 6 alternativas com escore correspondente que varia de zero a 5 pontos de acordo com a intensidade e gravidade da dor e comprometimento da coluna lombar. O trabalhador foi 
instruído a assinalar, em cada sessão, a alternativa que melhor representasse seu estado físico. O resultado final foi obtido em porcentagem por meio da fórmula: soma dos escores das sessões x $100 \div 50$, onde 50 representa o escore máximo possível. Os valores mais altos se referem à maior incapacidade do indivíduo.

O questionário de Roland-Morris é um instrumento composto por 24 alternativas a serem assinaladas com o objetivo de relatar as situações cotidianas e laborais que podem estar comprometidas pela lombalgia. Os sujeitos foram orientados a marcar as alternativas consideradas verdadeiras de acordo com a sua condição física. Quanto maior o número de itens assinalados, pior o estado de saúde do avaliado.

Em seguida, foi aplicado o teste de avaliação física Screening, proposto pela Polestar Education ${ }^{\circledR}$, para avaliação dos níveis de força dos músculos estabilizadores do tronco e da amplitude de movimento da coluna vertebral. Consiste em exame físico formado por 15 autoposturas executadas pelo avaliador e repetidas pelo examinado, que recebe uma pontuação de zero a 3 em cada uma das etapas. Para obtenção do escore final, todos os valores são somados e, em seguida, divide-se o resultado da soma por 15. Desta forma, foi mensurado o nível de condicionamento físico dos sujeitos, sendo zero referente a não tentativa de execução, 1 ao nível iniciante, 2 ao intermediário e 3 ao nível avançado.

As entrevistas e avaliações ocorreram individualmente, em cada posto de trabalho, no próprio horário de expediente e todas as etapas foram de responsabilidade de um único pesquisador, evitando assim alterações de execução e resposta. As informações coletadas foram devidamente protocoladas, arquivadas junto à pesquisadora responsável e submetidas à análise descritiva para expor os resultados em tabelas ou gráficos.

Para análise das variáveis qualitativas foi aplicado o teste Qui-quadrado ou o Exato de Fisher. Para testar a hipótese de normalidade dos dados foi aplicado o teste de Kolmogorov-Smirnov, e para análise comparativa das variáveis quantitativas foi aplicado o teste $t$ de Student. Para testar a suposição de homogeneidade das variâncias foi aplicado o teste de Levene. Os softwares utilizados foram o Excel 2000 e o SPSS v8.0 e todas as conclusões foram tomadas ao nível de significância de 5\%.

\section{RESULTADOS}

Os dados sugerem correlação significativa entre atividades laborais executadas na posição sentada e exis- tência de lombalgia $(p=0,001)$. Dos 146 funcionários sintomáticos, a prevalência de dor lombar crônica foi de $95,2 \%$, sendo maior nos funcionários com idade superior a 40 anos (Tabela 1), sedentários (Tabela 1) e que trabalham há mais tempo na instituição (Tabela 2). As variáveis sexo (Tabela 1), IMC (Tabela 2) e realização de pausas durante a jornada de trabalho (Tabela 1) não se comportaram como fator de risco para o aparecimento da sintomatologia.

A análise comparativa dos dados obtidos com a aplicação do exame físico revela menor prevalência de lombalgia entre os funcionários classificados como nível avançado $(\mathrm{p}<0,001)$. Os resultados da escala de dor e dos questionários de avaliação funcional descrevem a existência de limitações na realização de atividades profissionais e de vida diária nos indivíduos portadores de dor lombar (Gráfico 1).

Tabela 1 - Dor lombar em relação ao sexo, idade, prática de atividade física e realização de pausas durante a jornada de trabalho.

\begin{tabular}{lccc}
\hline Variáveis & Sim (\%) & Não (\%) & Valor de p \\
\hline Sexo & & & \\
$\quad$ Feminino & 64,2 & 35,8 & \\
$\quad$ Masculino & 56,9 & 43,1 & 0,248 \\
Faixa etária & & & \\
$\quad$ Até 40 & 49,4 & 50,6 & \\
$\quad 41$ a 50 & 69,4 & 30,6 & \\
$>50$ & 65,2 & 34,8 & 0,020 \\
Prática de atividade física & & & \\
$\quad$ Sim & 38,4 & 61,6 & \\
$\quad$ Não & 71,1 & 28,9 & $<0,001$ \\
Pausas durante a jornada & & & \\
de trabalho & & & \\
$\quad$ Sim & 52,9 & 47,1 & \\
$\quad$ Não & 63,3 & 36,7 & 0,178 \\
\hline
\end{tabular}

Tabela 2 - Dor lombar em relação ao índice de massa corporal e anos de trabalho na instituição.

\begin{tabular}{lcccc}
\hline & N & Média & Desvio & $\begin{array}{c}\text { Valor } \\
\text { de } p\end{array}$ \\
\hline $\begin{array}{l}\text { Índice de massa corpórea } \\
\quad \text { Com dor lombar }\end{array}$ & 146 & 26,2 & 4,2 & \\
$\quad$ Sem dor lombar & 93 & 25,4 & 4,1 & 0,164 \\
Tempo de trabalho & & & & \\
$\quad$ Com dor lombar & 146 & 18,9 & 10,2 & \\
Sem dor lombar & 93 & 16,2 & 10,5 & 0,049 \\
\hline
\end{tabular}




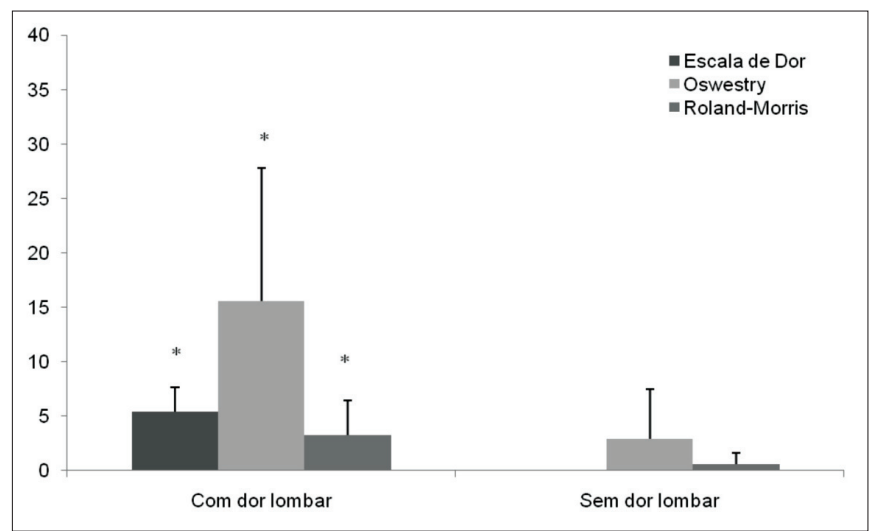

Gráfico 1 - Distribuição dos funcionários quanto a Escala de Dor e Questionários de Oswestry e Roland-Morris. $*(\mathrm{p}<0,001)$

\section{DISCUSSÃO}

O estudo identificou alta incidência de lombalgia entre os indivíduos que executam suas atividades laborais sentados. A postura sentada mantida por tempo prolongado pode gerar carência de flexibilidade muscular e de mobilidade articular, além de fadiga dos músculos extensores espinhais que, aliados, comprometem a estabilidade e o alinhamento da coluna vertebral. Tais distúrbios biomecânicos são considerados importantes fatores etiológicos para o desenvolvimento de dor lombar ${ }^{1,6}$.

Acredita-se que a postura sentada e o sedentarismo podem provocar alterações musculoesqueléticas, como a diminuição dos níveis de força e de amplitude de movimento $^{1,5,8}$, encontradas nos participantes da pesquisa.

O uso excessivo da cadeira pode ocasionar um encurtamento gradual dos músculos iliopsoas e isquiostibiais, diminuindo a mobilidade da articulação do quadril e inclinando para frente o segmento lombar da coluna vertebral ${ }^{1,6}$. Além disso, essa posição pode causar fadiga e sobrecarga nos elementos passivos articulares (cápsulas, ligamentos e discos intervertebrais), ocasionando dessensibilização dos mecanoceptores teciduais e consequente redução ou eliminação da força estabilizadora profunda, gerada principalmente pelo multifídio lombar e transverso abdominal ${ }^{9-12}$.

Tais alterações podem ser minimizadas ou evitadas pela prática regular de atividade física, uma vez que a incidência de lombalgia é menor em indivíduos ativos ${ }^{8,13}$, como sugerido através dos resultados do presente estudo. A prática de atividade física causa adaptações circulatórias e metabólicas benéficas para musculatura esquelética e tecidos conectivos, contribuindo para melhora da postura estática e dinâmica e redução do risco de lesões e incapacidades osteomusculares ${ }^{1,13}$.
Neste estudo, apesar dos portadores de lombalgia terem apresentado IMC mais elevado do que os indivíduos assintomáticos e serem, em sua maioria, mulheres, não se encontrou correlação estatisticamente significativa, provavelmente porque os valores de IMC encontrados não indicaram sobrepeso e, por se tratar de uma população com idade homogênea, a literatura relata que a correlação entre sexo feminino e dor lombar torna-se mais evidente com o avançar dos anos ${ }^{8,14}$.

Estudos revelam que a sobrecarga mecânica ocasionada pela dupla jornada de trabalho a qual as mulheres são submetidas parece estabelecer uma associação entre sexo feminino e a sintomatologia dolorosa ${ }^{8,13,14}$. O excesso de gordura no abdômen dos indivíduos com sobrepeso é apontado como fator de risco para o desenvolvimento de lombalgia por deslocar o centro de gravidade corporal $1^{8,14}$.

A ausência de momentos de repouso pode agravar o quadro clínico dos funcionários portadores de lombalgia ${ }^{6,13}$, interferindo diretamente na execução das atividades de vida diária e profissional ${ }^{1,3}$, como já pode-se observar pela aplicação dos questionários de Oswestry $^{3}$ e Roland-Moris ${ }^{3}$. Pesquisa com metodologia semelhante ${ }^{3}$ corrobora com o comportamento encontrado neste estudo ao descrever uma possível associação entre limitação funcional e dor lombar crônica.

Considerando que o trabalho executado na postura sentada parece atuar como fator nocivo, provocando um desequilíbrio mecânico dos componentes da coluna vertebral ${ }^{5}$, seria interessante a realização de medidas preventivas para minimizar o quadro sintomatológico; e, principalmente, evitar o risco de surgimento de distúrbios musculoesqueléticos nos indivíduos saudáveis. É importante o reconhecimento do ambiente de trabalho antes da implantação de qualquer programa de intervenção, seja ele preventivo e/ou terapêutico, além do acompanhamento regular dos projetos executados para estimular mudanças de comportamento em longo prazo ${ }^{15}$.

\section{CONCLUSÃO}

Os resultados sugerem uma provável associação entre dor lombar e atividades laborais na posição sentada e alertam para a necessidade de cuidados com a saúde da coluna no ambiente de trabalho.

\section{REFERÊNCIAS}

1. Reis PF, Moro ARP, Contijo LA. A importância da manutenção de bons níveis de flexibilidade nos trabalha- 
dores que executam suas atividades laborais sentados. Rev Prod On Line 2003;3(3).

2. Almeida ICGB, Sá KN, Silva M, et al. Prevalência de dor lombar crônica na população da cidade de Salvador. Rev Bras Ortop 2008;43(3):96-102.

3. Pereira JE, Pinto MC, Souza RA. Prevalência de lombalgias em transportadores de sacos de café. Rev Motriz 2006;12(3):229-38.

4. Kovacs FM, Abraira V, Zamora J, et al. Correlation between pain, disability, and quality of life in patients with common low back pain. Spine 2004;29(2):206-10. 5. Toscano JJO, Egypto EP. A influência do sedentarismo na prevalência de lombalgia. Rev Bras Med Esporte 2001;7(4):132-7.

6. Sacco ICN, Aliberti S, Queiroz BWC, Pripas D, Kieling I, Kimura AA et al. A influência da ocupação profissional na flexibilidade global e nas amplitudes angulares dos membros inferiores e da lombar. Rev Bras Cineantropom Desempenho Humano 2009; 11(1):51-8.

7. Horng YS, Hwang YH, Wu HC, et al. Predicting health-related quality of life in patients with low back pain. Spine 2005;30(5):551-5.

8. Silva MC, Fassa AG, Valle NC. Chronic low back pain in a Southern Brazilian adult population: prevalence and associated factors. Cad Saude Publica 2004;20(2):377-85. 9. Dankaerts W, O'Sullivan P, Burnett A, et al. Altered patterns of superficial trunk muscle activa- tion during sitting in nonspecific chronic low back pain patients: importance of subclassification. Spine 2006;31(17):2017-23.

10. Hides J, Wilson S, Stanton W, et al. An MRI investigation into the function of the transversus abdominis muscle during "drawing-in" of the abdominal wall. Spine 2006;31(6):E175-8.

11. França FJR, Burke TN, Claret DC, Marques AP. Estabilização segmentar da coluna lombar nas lombalgias: uma revisão bibliográfica e um programa de exercícios. Fisioter Pesq 2008;15(2):200-6.

12. Panjabi MM. A hypothesis of chronic back pain: ligament subfailure injuries lead to muscle control dysfunction. Eur Spine J 2006;15(5):668-76.

13. Maciel ACC, Fernandes MB, Medeiros LS. Prevalência e fatores associados à sintomatologia dolorosa entre profissionais da indústria têxtil. Rev Bras Epidemiol 2006;9(1):94-102.

14. Azevedo JVS, Silva JRL, Ribeiro DCL. Relação entre lombalgia e sobrepeso em praticantes de atividade física. ConScientiae Saúd 2008;7(4):471-5.

15. Martins MRI, Foss MHDA, Santos Júnior R, et al. A eficácia da conduta do Grupo de Postura em pacientes com lombalgia crônica. Rev Dor 2010(2):116-21.

Apresentado em 11 de julho de 2011.

Aceito para publicação em 25 de agosto de 2011. 